\title{
NEW COLD FET I-Q LINEAR VECTOR MODULATOR TOPOLOGY
}

\author{
Cyrille Boulanger, Luc Lapierre, Francis Gizard \\ CNES 18, Av E. Belin 31401 Toulouse cedex. \\ Phone : (33) 5.61.27.37.85 Fax : (33) 5.61.28.26.13 $\quad$ E-Mail : cyrille.boulanger@cnes.fr
}

\section{I- ABSTRACT}

This paper presents the design, manufacture and test of a new cold FET monolithic I-Q vector modulator. The circuit has been developed in 8-8.4 GHz $X$ band but can be transposed at any frequency range. The innovation is that the IF data signals are applied to the FET gates. The use of cold FETs allows achieving a $0.8 \mathrm{~dB}$ and $6^{\circ}$ accuracy with only one voltage tuning on the whole bandwidth and the $-25^{\circ} \mathrm{C}$ to $+50^{\circ} \mathrm{C}$ temperature range. There is no DC consumption for the microwave modulator. The accuracy achieved at this first run is yet compatible with new coding and modulating systems and we have investigated several ways to improve the design.

\section{II- DESCRIPTION :}

In order to improve the efficiency of transmitting information through power and bandwidth limited channels that are requirements of space-borne systems, new coding and modulating systems are under studies using non constant envelope signals like Square Raised Cosine Modulation or Quadrature Amplitude Modulation.

I-Q linear vector modulators are then required and have been published yet [1] with very good results but with quite complex structure and high consumption. We propose a new structure based on the topology we have developed in the past [2] to design a QPSK in a commutation mode. This topology is based on the use of cold FETs and needs only one voltage tuning to optimise the performances in frequency. It has been patented [3].

In the Earth Observation frame, CNES has developed a new telemetry payload achieving quality losses less than $1.6 \mathrm{~dB}$ from theory at 50 Mbauds, 2bits $/ \mathrm{s} / \mathrm{Hz}$ with $\mathrm{E}_{\mathrm{b}} / \mathrm{N}_{0}=10^{-7}$ in direct 8 PSK SRC with a 0.35 roll off, Trellis Coded Modulation using this new X band I-Q modulator.
The I-Q vector modulator is represented on figure 1 and 2. We have designed an I-Q vector modulator in the UMS PH25 PHEMT process. It's composed by an input cell splitting the LO carrier into two $90^{\circ}$ phase shifted signals. These signals drive two cold FET $180^{\circ}$ BPSK modulator and then are summed at the output. Both modulators are driven by two complementary data signals.

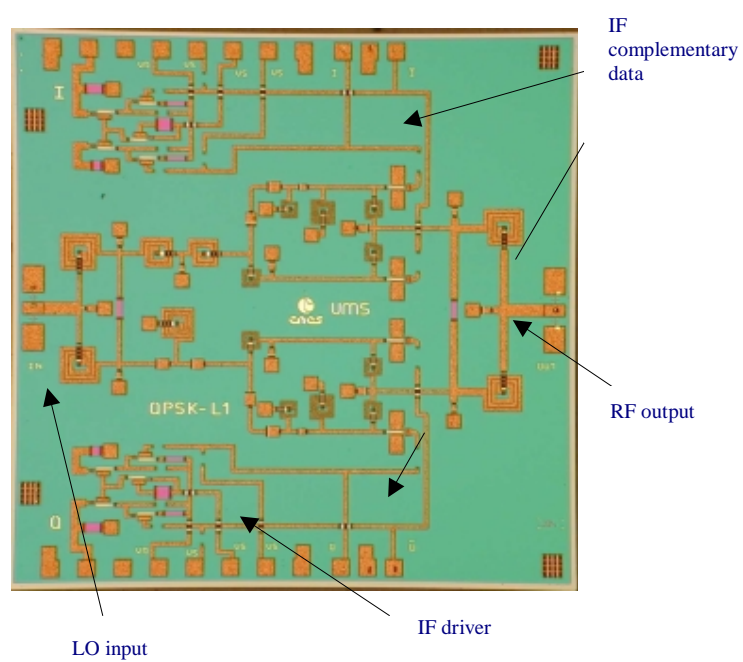

Fig 1: chip or the moaulator with IF drivers, $3 \times 3 \mathrm{~mm}^{2}$

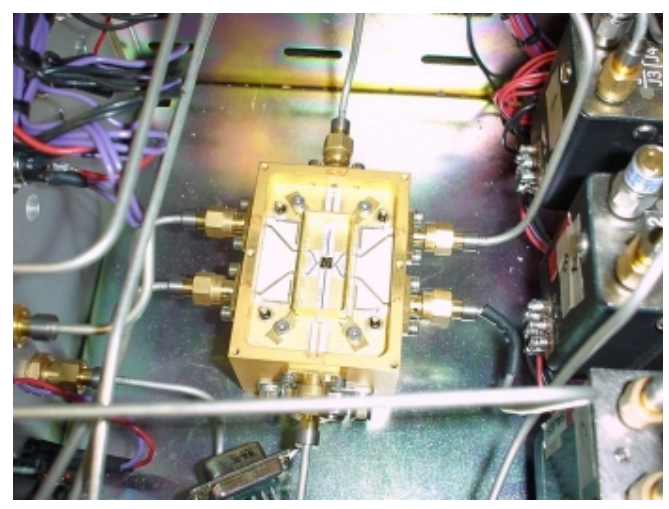

Fig 2 : chip integrated in the whole testbench

The innovation is that the IF data signals are applied to the gates of the FETs and the LO and $\mathrm{RF}$ are on the drain. The isolation between high frequencies and IF is given by the unilaterality of 
the transistor. Thus there is no need of filters. Another advantage is that the conversion losses of the cold FET in this configuration are independent of the LO frequency. This point has been simulated on a single cold transistor alone and doesn't take into consideration losses in the passive elements. Usually cold FETs are used in commutation, taking benefit of the $\mathrm{R}_{\mathrm{ds}}$ value differences between onstate $\left(\mathrm{V}_{\mathrm{gs}}=0 \mathrm{~V}\right)$ and off-state $\left(\mathrm{V}_{\mathrm{gs}}<\mathrm{V}_{\mathrm{p}}\right)$. In fact we have noticed that we can obtain a linear behaviour of this component by reducing the excursion of the data signals.

The new topology we propose with cold FETs demonstrates the capability to design an I-Q modulator with no DC consumption and only one tuning voltage. This tuning consists of defining the center voltage $V_{c}$ of the data signals near the pinch-off voltage where there is the knee on the equivalent resistance as seen on the figure 3 .

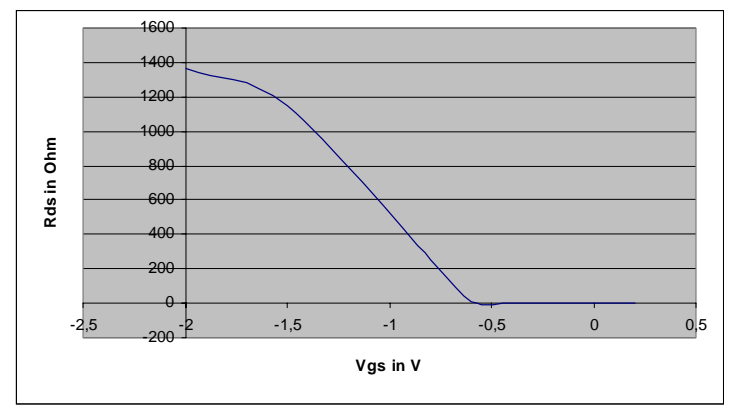

Fig $3: R_{d s}=f\left(V_{g s}\right)$ for a cold FET

Once this is done there's no more tuning to use this circuit over the full 7.8-8.6 GHz bandwidth and over the $-25 /+50^{\circ} \mathrm{C}$ temperature range (For our need, we just present the 8-8.4 GHz bandwidth). In order to simplify the interface between the microwave modulator and the data processing systems we have included on the chip drivers able to adapt the data coming from the logic circuits to the microwave modulator. From a single data centered on a 0 voltage, this driver generates two complementary signals centered on the optimal voltage. The structure of the IF drivers is a simple differential amplifier with a second stage generating the $\mathrm{V}_{\mathrm{c}}$ offset. This basic circuit can be improved, to be compatible with existing logic families.

The circuit was designed to be fed through the IF drivers or directly by formatted complementary data.

\section{III- RÉSULTS :}

The on wafer results have been obtained by static states measurement and encapsulated circuit was characterised by spectrum measurements. These results are presented here.

The spectrum measurement consists of applying two sine waves on the I and Q modulators with a $90^{\circ}$ phase shift on the I and Q inputs. We generate all the harmonic products of LO and IF. The useful signal is the LO-IF or LO+IF. We characterise the modulator accuracy by the carrier and the corresponding image frequency rejections.

Phase states errors are deduced from the expressions above defined in [1].

$$
\begin{aligned}
& \delta A \leq 20 \log (1+\text { Crej }+\sqrt{2} \cdot \text { Brej }) \\
& \delta \theta \leq A r c \tan (\text { Crej }+\sqrt{2} \cdot \text { Brej })
\end{aligned}
$$

$\mathrm{C}_{\mathrm{rej}}$ is the carrier rejection, $\mathrm{B}_{\mathrm{rej}}$ is the image frequency rejection.

We have obtained a $\mathbf{0 . 8} \mathbf{~ d B}$ and $6^{\circ}$ of phase state accuracy and a $6 \mathrm{~dB}$ RF linear behaviour with respect to the IF signals with no DC consumption. Input and output matchings are best than $18 \mathrm{~dB}$ on the whole bandwidth and state switching does not generate impedance modification.

These performances are far from I-Q linear vector modulator state of the art but are sufficient for very performing coding systems like Trellis Coded Modulation which are not very sensitive to the microwave modulator accuracy.

The useful range is limited by the RF signal compression for high data signal level (cf figure 4), and by the accuracy on amplitude and phases for low data signal level. (cf figure 5).

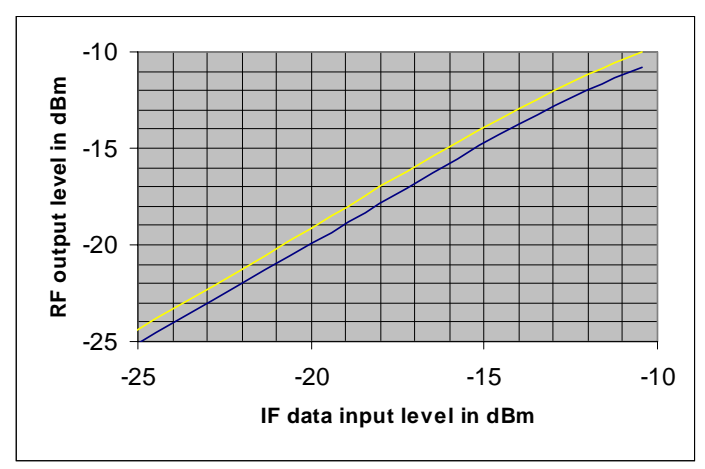

Fig 4 : RF Pout versus IF Pin at $-25^{\circ} \mathrm{C}$ and $+50^{\circ} \mathrm{C}$ 
This circuit needs a high LO level $(5 \mathrm{dBm}$ in our case) and achieves $15 \mathrm{~dB}$ of $\mathrm{LO}$ to RF conversion losses which was expected for a non biased transistor circuit. The linearity is obtained up to $10 \mathrm{dBm}$ IF input level. The use of cold FETs induces a very high manufacture efficiency and a very low sensitivity to temperature as it can be seen on the graphs of figure 5 .

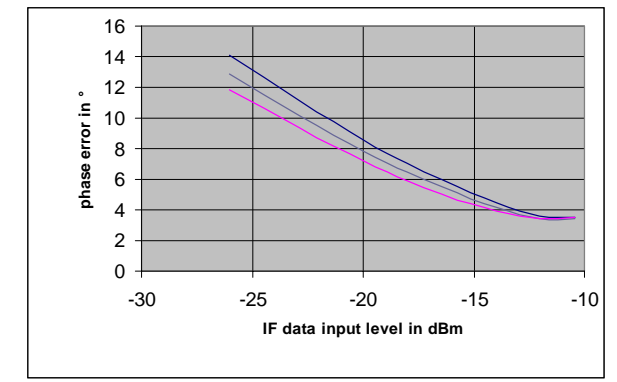

phases errors at $8,8.2$ and $8.4 \mathrm{GHz}$ at $+50^{\circ} \mathrm{C}$

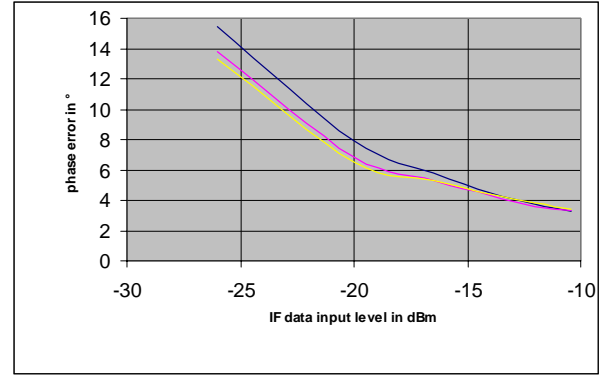

phases errors at $8,8.2$ and $8.4 \mathrm{GHz}$ at $-25^{\circ} \mathrm{C}$

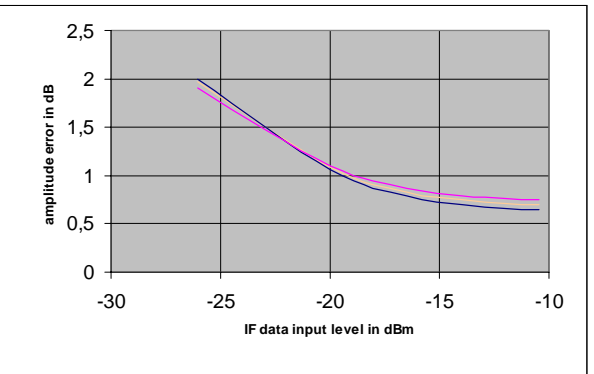

amplitudes errors at $8,8.2$ and $8.4 \mathrm{GHz}$ at $+50^{\circ} \mathrm{C}$

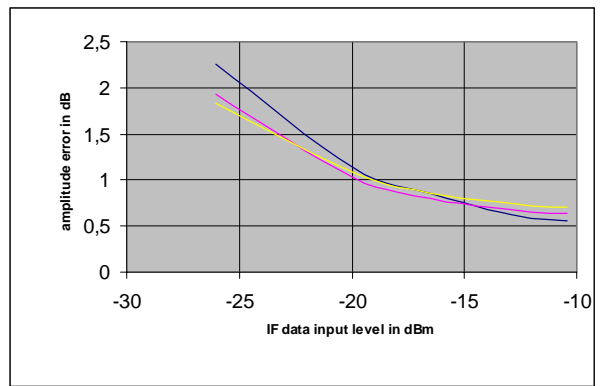

amplitudes errors at $8,8.2$ and $8.4 \mathrm{GHz}$ at $-25^{\circ} \mathrm{C}$

Fig 5 : performances in temperature and frequency
In the Earth Observation frame, CNES has developed a new telemetry payload achieving a quality losses from theory :

less than $1.6 \mathrm{~dB}$ at 50 Mbauds

2bits/s/Hz

with $\mathrm{E}_{\mathrm{b}} / \mathrm{N}_{0}=10^{-7}$

in direct 8 PSK SRC (roll off $=0.35$ )

Multidimensional Trellis Coded Modulation

\section{IV- IMPROVEMENTS}

Measurements have shown that the image frequency rejection is to be improved. This is directly correlated to the $90^{\circ}$ input power divider, the carrier rejection being mainly correlated to the $180^{\circ}$ BPSKs accuracy. By using a Lange coupler, for example, it could be possible to achieve a best accuracy on the full bandwidth. By simulations we have seen that using bigger transistors in the BPSK modulators improves the carrier rejection and using a parallel LC feedback loop on these FETs is a possible way to increase this circuit linearity range.

\section{V- CONCLUSION}

We have demonstrated the possibility to design a very simple non biased $\mathrm{X}$ band $\mathrm{I}-\mathrm{Q}$ vector modulator with sufficient accuracy for new coding and modulating systems. Solutions to improve the accuracy are identified and will be implemented in a future Ka band version.

\section{REFERENCES}

[1] FLM Van den Bogaart, R Pyndiah, "a 10-14 GHz linear MMIC vector modulator with less than $0.1 \mathrm{~dB}$ and $0.8^{\circ}$ amplitude and phase error"

1990 IEEE MTT-S Digest

[2]. A. Primerose, J. Graffeuil, L. Lapierre, J. Sombrin, J.C. Lalaurie, J. Larroque. «High Bit Rate Four Phase MMIC Remodulation Demodulator and Modulator», GaAs 92 ESA/ESTEC Symposium, NOORDWIJK-The NETHERLANDS.

[3] C. Boulanger, L. Lapierre,

demande de brevet $\mathrm{n}^{\circ} \mathrm{PCT} / \mathrm{FR} 99 / 03276$ «procede de modulation analogique et circuit integre monolithique a étage mélangeur comprenant des transistors à effet de champ non polarisés" » 